\title{
Método cualitativo para la determinación del estatus de conservación de Bulnesia sarmientoi (Zygophyllaceae) en Paraguay con fines de exportación
}

\author{
G. CÉSPEDES ${ }^{1,2}$, F. MERELES ${ }^{2} \&$ R. M. NAVARRO-CERRILLO ${ }^{3}$ \\ ${ }^{1}$ Asociación Etnobotánica Paraguaya (AEPY) y Consejo Nacional de Ciencia y Tecnología (PRONII-CONACYT), \\ Ecuador, 450, PY-2420 Lambaré, Paraguay \\ ${ }^{2}$ Centro para el Desarrollo de la Investigación Científica (CEDIC), Laboratorios Díaz-Gill, Asunción-Paraguay \& Fundación Moisés \\ Bertoni, Manduvira 635, PY-1255 Asunción, Paraguay y Consejo Nacional de Ciencia y Tecnología (PRONII-CONACYT) \\ ${ }^{3}$ Departamento de Ingeniería Forestal-DendroLab-Universidad de Córdoba, Campus Universitario Rabanales, \\ ctra. Nacional IV, km 396, ES-14014 Córdoba, España
}

ORCID iD. G. CÉSPEDES: https://orcid.org/0000-0001-8811-048X, F. MERELES: http://orcid.org/0000-0002-2866-2204, R. M. NAVARRO-CERRILLO: http://orcid.org/0000-0003-3470-8640

Autor para correspondencia: G. Céspedes (gloriacespedes@etnobotanica.org.py)

Editor: A. Susanna

Recibido 31 marzo 2016; aceptado 20 diciembre 2017; publicado on line 29 agosto 2018

\begin{abstract}

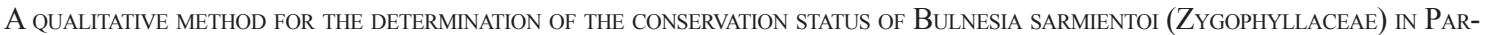
AGUAY FOR EXPORTATION PURPOSES.- A qualitative analysis of the reasons why the species Bulnesia sarmientoi is included in Appendix II of the Convention on International Trade in Endangered Species of Wild Fauna and Flora (CITES) has been performed using the methodology proposed by the IUCN. This analysis was supplemented with information related to derivative products commercialization and data concerning growth, phenology, ecology and deforestation in the area occupied by the species. Analyses showed several information gaps, especially quantitative data, to establish the species conservation status. Results indicate that Bulnesia sarmientoi populations are under severe threat such as accelerated habitat loss and unsustainable timber harvesting and derivate products. The study concludes that quantitative population studies should be conducted to determine export quotas, without which it will not be possible to ensure the continuity of the populations of this species.
\end{abstract}

Key words: Bulnesia sarmientoi; conservation; Paraguayan Chaco; qualitative analysis.

\begin{abstract}
Resumen
Método cualitativo para la determinación del estatus de conservación de Bulnesia sarmientoi (ZygophyllaCeae) en ParaGUAY CON FINES DE EXPORTACIÓN.- Se realiza un análisis cualitativo, mediante el uso de la metodología propuesta por la UICN, de las causas por las que la especie Bulnesia sarmientoi se encuentra incluida dentro del Apéndice II de la Convención Internacional para el Tráfico de la Vida Silvestre (CITES). Estos datos se complementan con información procedente de la comercialización de los productos derivados, así como de otros datos referentes al crecimiento, la fenología, la ecología y la deforestación del área ocupada por esta especie. Los análisis muestran varios vacíos de información, especialmente de datos cuantitativos, para la determinación de su estado de conservación. Los resultados indican que las poblaciones de Bulnesia sarmientoi se encuentran sometidas a fuertes amenazas, como la pérdida acelerada de su hábitat y a la explotación no sostenible de su madera y de los productos derivados. El estudio concluye que se deberán realizar estudios poblacionales cuantitativos tendentes a la obtención de cupos de exportación, sin los cuales no se podrá garantizar la supervivencia de las poblaciones de la especie.
\end{abstract}

Palabras clave: Bulnesia sarmientoi; Chaco paraguayo; conservación; estudios cualitativos.

Cómo citar este artículo / Citation

Céspedes, G., Mereles, F. \& Navarro-Cerrillo, R. M. 2018. Método cualitativo para la determinación del estatus de conservación de Bulnesia sarmientoi (Zygophyllaceae) en Paraguay con fines de exportación. Collectanea Botanica 37: e006. https://doi.org/10.3989/collectbot.2018.v37.006

Copyright

(C) 2018 CSIC. This is an open-access article distributed under the terms of the Creative Commons Attribution 4.0 International (CC BY 4.0) License. 


\section{INTRODUCCIÓN}

Bulnesia sarmientoi Lorentz ex Griseb., el "palo santo", es una de las especies leñosas endémicas del Gran Chaco Americano. Es un árbol de porte esbelto, de floración muy llamativa, caracterizado por su lento crecimiento y por el olor y calidad de su madera, lo que la convierte en una de las especies más valoradas del bosque chaqueño. Históricamente, es uno de los árboles más utilizados en la región; sin embargo, hay una ausencia generalizada de estudios cuantitativos que pudieran dar pautas claras para el manejo y exportación de la especie, motivo por el cual se ha desembocado en una situación poco clara de su estatus real de conservación.

Esta especie se distribuye desde el sudeste de Bolivia (departamentos de Oruro, Santa Cruz y Tarija hasta $17^{\circ} \mathrm{S}$ ), centro-oeste-este de Paraguay (departamentos Presidente Hayes, Boquerón y Alto Paraguay), sectores limítrofes de Brasil (Estado de Mato Grosso) y norte de Argentina, donde alcanza su límite austral a los $25^{\circ} \mathrm{S}$ (Provincias de Chaco, Salta, Formosa y, marginalmente, Santiago del Estero).

Es un árbol de 8 a $25 \mathrm{~m}$ de altura, de copa rala, estrecha, redondeada en el ápice, ramas ascendentes, con entrenudos de $2-3 \mathrm{~cm}$ de longitud y nudos ensanchados con anillos. Tronco recto, corto, a veces acanalado, con un diámetro a la altura el pecho (DAP, a 1,30 m) de 30-70 cm, corteza externa parda, áspera y verrugosa, con grietas irregulares poco profundas que forman placas irregulares a lo largo del tallo; la corteza interna es resinosa, de color amarillento con gránulos diminutos de color anaranjado (López et al., 1987).

La madera del "palo santo" tiene un alto valor comercial, por su gran resistencia y por ser prácticamente imputrescible, debido al aceite esencial que contiene, siendo una de las maderas más duras del mundo (densidad: $0,92-1,1 \mathrm{~g} / \mathrm{cm}^{3}, 1100-1280$ $\mathrm{kg} / \mathrm{m}^{3}$, López et al., 1987). La especie tiene utilidad principalmente forestal y se emplea para la elaboración de muebles finos, suelos de parqué, tornería, y fabricación de artículos decorativos y bujes navales (Arenas, 1981; Schmeda-Hirschmann, 1993; Mereles \& Degen, 1997a; Scarpa, 2000). Localmente se utiliza en la fabricación de postes. También se aprovecha como leña, ya que se enciende fácilmente y produce un humo con una gran fragancia; además se emplea en medicina popular (Arenas, 1983;
Amat \& Yajía, 1991; Filipov, 1997; Salvat et al., 2004; Pin et al., 2009). El aceite esencial es muy apreciado (Rempel, 2003) y se exporta para su utilización en la industria cosmética y de perfumería (Rodilla, 2011).

La especie alcanza el estado adulto aproximadamente a los 20 años (Brack \& Weik, 1994). Las semillas se dispersan por autocoria y anemocoria y, aunque la producción de frutos y semillas es abundante (López et al., 1987), la tasa de germinación es baja. Estudios poblacionales realizados en $\mathrm{Pa}$ raguay revelan que la regeneración por semillas es mínima, y en algunos casos nula, y que la mayor parte de la capacidad de regeneración es a través de nuevos vástagos que brotan de las raíces en algunos ejemplares adultos en pie (Mereles \& Pérez de Molas, 2007). Hay varios factores que influyen en la capacidad de regeneración vegetativa, como las condiciones del suelo, la temperatura y la necesidad de agua, entre otros.

Bulnesia sarmientoi crece en los bosques xerófitos del Chaco; según Mereles (2005), se desarrolla sobre suelos arcillosos, muy estructurados, duros cuando están secos, y plásticos cuando están húmedos, de salobres a salados, y con capa freática salobre a salada por encima de los seis metros de profundidad. En este ambiente, es la especie dominante en el estrato superior del bosque, frecuentemente asociada con Ziziphus mistol Griseb., Maytenus vitis-idaea Griseb., Grabowskia duplicata Arn. y Trithrinax schyzophylla Drude (Mereles \& Pérez de Molas, 2007; Mereles, 2014). También se la encuentra en forma aislada dentro del bosque xerófito, acompañando a Aspidosperma quebracho-blanco Schltdl., Sarcotoxicum salicifolium Cornejo \& Iltis, Cynophalla retusa Cornejo \& Iltis, Tabebuia nodosa Griseb. y Stetsonia coryne (Salm-Dyck) Britton \& Rose, entre otras. Según Viveros Cabaña (2005), los suelos donde mejor se adapta la especie son los cambisoles (fracción de arcilla del 30\% y limosa bastante alta) y luvisoles (los más abundantes del Chaco central, arenosos pasando por textura limosa hasta arcillosa en profundidades de hasta $70 \mathrm{~cm}$, ricos en $\mathrm{P}, \mathrm{Mg}$ y $\mathrm{K}$ ), y eventualmente los gleysoles (saturados durante periodos prolongados, a partir de los $50 \mathrm{~cm}$, generalmente con un porcentaje entre el 50 y el $80 \%$ de arcilla, en algunos casos con alto contenido de sal). Otros autores mencionan la preferencia de la especie por los suelos arcillosos no muy bien drenados (Navarro, 1997). En Paraguay 
la especie ocupaba, en la década de 1980, una superficie aproximada de $37.000 \mathrm{~km}^{2}$, y en Argentina $25.000 \mathrm{~km}^{2}$ (CITES, 2010), no encontrándose información sobre la situación en Bolivia. Aunque no se tengan datos exactos de nuevos censos de la especie, se considera que la modificación del hábitat por la deforestación para el cambio del uso del suelo en el Chaco Paraguayo y la extracción indiscriminada de la especie han mermado mucho sus poblaciones (Viveros Cabaña, 2005).

El Paraguay es, en la actualidad, el mayor exportador de madera y de los derivados del "palo santo". La explotación excesiva de su madera, unida a ser una especie de crecimiento muy lento y de distribución en áreas muy reducidas, ha hecho que la especie haya adquirido un estatus especial de conservación nacional donde se especifica que la misma no puede ser explotada ni industrial ni comercialmente, a menos que cuente con un plan de manejo aprobado por las autoridades competentes. Sin que hubieran sido conocidos estudios poblacionales en Paraguay, la especie fue considerada para su inclusión dentro de los apéndices de la Convención Internacional para el Tráfico de la Vida Silvestre (CITES) debido a su similitud con otra, Guaiacum sanctum L., de la misma familia e incluida dentro del Apéndice II desde el año 2003 (López-Toledo et al., 2008). Esto implicó la disminución de la disponibilidad de los productos derivados de la misma en el mercado internacional y el aumento de la demanda del "palo santo" en Paraguay, con la consecuente preocupación de la comunidad científica paraguaya quienes, considerando las amenazas a la especie, elevaron una propuesta de inclusión de la misma al Apéndice III de la Convención CITES. En la actualidad, la especie se encuentra en el Apéndice II de la Convención CITES con anotación \#11, que designa trozas, madera aserrada, láminas de chapa de madera, madera contrachapada, polvos y extractos.

Las medidas tomadas por parte de las autoridades paraguayas con respecto a la protección del "palo santo" se iniciaron con el Decreto $\mathrm{N}^{\circ}$ $18.105 / 1993$, que restringe la corta y el aprovechamiento del "palo santo", y que fue seguido por otros desarrollos normativos (Resolución 128/03), que establecieron un DAP mínimo de corta de 30 $\mathrm{cm}$, así como la superficie máxima de las áreas de desmonte que contengan "palo santo" $(<100$ ha en forma continua). En el año 2004, la Secretaría del
Ambiente (SEAM) publicó la lista de "elementos especiales", donde se incluye $B$. sarmientoi con rango de categoría N2N3, lo que implica que la especie está en peligro en el país debido a su rareza (6-20 localizaciones, pocos individuos o hectáreas ocupadas por la especie). En el año 2006, la especie fue incluida dentro de la lista de especies amenazadas de la SEAM (Resolución N $\mathrm{N}^{\circ}$ 524/06; Resolución $\mathrm{N}^{\mathrm{o}}$ 2243/06; Resolución $\mathrm{N}^{\mathrm{o}} 2531 / 06$ ), donde se establece que las especies no podrán ser explotadas ni industrial ni comercialmente a excepción de las que dispongan de planes de manejos aprobados por la autoridad competente y que cuente con sus correspondientes Licencias Ambientales.

En cuanto al control sobre el comercio de la especie y sus derivados, en el año 2006 se sancionó la Resolución $\mathrm{N}^{\mathrm{o}} 729$ que prohíbe la exportación de cilindros torneados y parafinados de maderas de la misma. Ese mismo año, Paraguay presentó la propuesta de inclusión de la especie dentro del apéndice II de la CITES, durante la $16^{\circ}$ Reunión del Comité de Flora de CITES. Sin embargo, al siguiente año, se decidió suspender la vigencia de esta Resolución a través de otra, la No $473 / 07$. A pesar de las reglamentaciones establecidas respecto a los mecanismos de control y aprovechamiento de la especie se decidió, a través de la Resolución $\mathrm{N}^{\mathrm{o}} 797 / 07$, suspender temporalmente la exportación de productos forestales de maderas de "palo santo", exceptuándose de la misma aquellos productos terminados, legalmente amparados para la exportación y debidamente demostrados los compromisos contractuales asumidos con anterioridad. A través de la Resolución N ${ }^{\circ} 1105 / 07$, se autorizó la exportación únicamente para productos manufacturados de la especie, con previa presentación de la lista de proveedores y su correspondiente ubicación. Además, se comunica que el aprovechamiento se realizará en forma selectiva y racional a través de planes de manejo aprobados por la autoridad de aplicación. Adicionalmente, queda prohibida la quema de subproductos forestales resultantes de la ejecución de los planes de manejo autorizados tales como leña, carbón, postes, esencia, rollos para madera u otros usos.

Sucesivas irregularidades fueron detectadas en Paraguay por el Ministerio Público en cuanto a la gestión de entes públicos respecto al traslado de la madera, documentaciones requeridas para el control de la exportación de productos derivados de la 
especie, cantidad exacta, etc., en el ámbito de las competencias de la Dirección General de Aduanas, el Instituto Forestal Nacional y el Ministerio de Industria y Comercio, encargados de las funciones mencionadas durante el ejercicio fiscal 2006-2007. Respecto a los datos de comercio internacional, la Red de Inversiones y Exportaciones del Paraguay (REDIEX), registró en el año 2010 la exportación de 1248,4 toneladas de productos derivados de "palo santo" y en el 2011 de 984,85 toneladas; sin embargo, ese mismo año, World Conservation Monitoring Centre (WCMC-ONU), registró solamente la exportación de 41,596 toneladas, lo que hace una diferencia de más de 1000 toneladas entre los datos proveídos por el ente nacional y el internacional para 2010 y 2011.

En este contexto, las poblaciones paraguayas de "palo santo" están sufriendo una importante pérdida de hábitat debido al cambio de uso de suelo forestal (1.311.130 ha de bosque chaqueño deforestado entre los años 2010 y 2014 según Guyra Paraguay, 2014); la ganadería, la explotación no regulada de su madera y los vacíos de información relacionados con el estado de las poblaciones, la dinámica natural de las mismas y los agentes perturbadores, son otros causantes de la vulnerabilidad de la especie (M. Sanjurjo, 2011, com. pers.).

Considerando lo expuesto, este trabajo tiene por objetivo determinar cualitativamente, a partir los datos disponibles, el estado de conservación de $B$. sarmientoi en Paraguay, aportando una información preliminar para la elaboración de un Dictamen de Extracción No Perjudicial (sensu CITES) para su exportación, así como realizar recomendaciones para mejorar el manejo de la especie en el país.

\section{MATERIAL Y MÉTODOS}

\section{Área de estudio}

Los datos poblacionales utilizados en este trabajo, las observaciones interespecíficas y el estado de conservación del hábitat se obtuvieron de la propuesta de inclusión de "palo santo" en el apéndice II (Mereles \& Pérez de Molas, 2007), de dos parcelas de una hectárea localizadas en el centro de la región chaqueña $\left(22^{\circ} 45^{\prime} 32,2^{\prime \prime} \mathrm{S}, 61^{\circ} 07^{\prime} 03,7^{\prime \prime}\right.$ W y $23^{\circ} 22^{\prime} 01^{\prime \prime} \mathrm{S}, 60^{\circ} 28^{\prime} 20,8^{\prime \prime} \mathrm{W}$ ) y del monitoreo de la deforestación del Chaco (Guyra Paraguay,
2014). Los datos de distribución general (ver mapa de distribución de Bulnesia sarmentoi; Fig. 1) se obtuvieron de las colecciones depositadas en los herbarios de la Facultad de Ciencias Químicas de la Universidad Nacional de Asunción (FCQ), del Museo Nacional de Historia Natural del Paraguay (PY) y de la base de datos del Missouri Botanical Garden (http://www.tropicos.org).

\section{Fuentes de información}

Los datos biológicos sobre B. sarmientoi (crecimiento, ecología, fenología), así como aquellos relacionados con los usos y el comercio de la especie se obtuvieron de publicaciones científicas (Cozzo, 1948; Hunzicker, 1980; Arenas, 1981, 1983; Palacios, 1984; Mereles \& Degen, 1993, 1994, 1997a, $b$; Degen \& Mereles, 1996, 1997; Schulz, 1997; Giménez \& Moglia, 2003; Friesen Ratzlaff, 2004; Ulmke \& August, 2004; Alonso \& Desmarchelier, 2005; Tortorelli, 2009; Arambarri et al., 2011; Céspedes, 2011; Palacios \& Mom, 2012; Galeano, 2014), bases de datos e informes de la Convención CITES. Adicionalmente aportaron datos sobre el manejo, la comercialización y la exportación de la especie, la Federación Paraguaya de Madereros (FEPAMA), la Red de Inversiones y Exportaciones (REDIEX), el Banco Central del Paraguay (BCP), el Instituto Forestal Nacional (INFONA) y Asociación Latinoamericana de Integración (ALADI). Finalmente se realizaron entrevistas a autoridades nacionales relacionadas como la Autoridad Científica CITES en la Secretaría del Ambiente (SEAM), el INFONA y profesionales forestales nacionales (Céspedes, 2011).

\section{Listado de verificación para ayudar a formular dictámenes de extracción no perjudicial}

La valoración del estatus de conservación de la especie se hizo mediante el método denominado Checklist to assist in making non-detriment findings for Appendix II exports (Rosser \& Haywood, 2002). Dicho método se basa en la utilización de datos cualitativos, fundamentando las dificultades en el desarrollo de criterios "duros" para el uso sostenible, aplicables a una gama amplia de táxones y a ecosistemas diversos. Consiste en una lista que ayuda a identificar los factores necesarios para formular un Dictamen de Extracción No Perjudicial 
o "cuotas" (cantidad máxima de extracción de la especie en su medio). Es el método empleado por la Convención CITES; y ayuda a las Autoridades Científicas de los países miembros de la Convención a comprender los puntos fuertes y débiles de la información que existe a su disposición. La metodología propuesta organiza la información de manera gráfica, y resume de forma sencilla la situación de la especie, lo que permite comparar dictámenes de extracción no perjudicial con los de otros países, para especies similares o artículos similares, que son objetos de comercio. El método incluye dos etapas:
1. Análisis de los aspectos relacionados con la cosecha, la extracción, la biología, el control, la gestión, la vigilancia, los incentivos y la protección de la especie. Consiste en esquematizar la información disponible en una tabla-guía elaborada exclusivamente para especies vegetales, donde se colocan los datos obtenidos en las filas correspondientes a cada tema.

2. Evaluación de los resultados obtenidos sobre la investigación de la historia natural y la gestión a nivel nacional de la especie. Los parámetros que se tienen en cuenta son los siguientes: formas de vida, potencial de regeneración, eficacia de

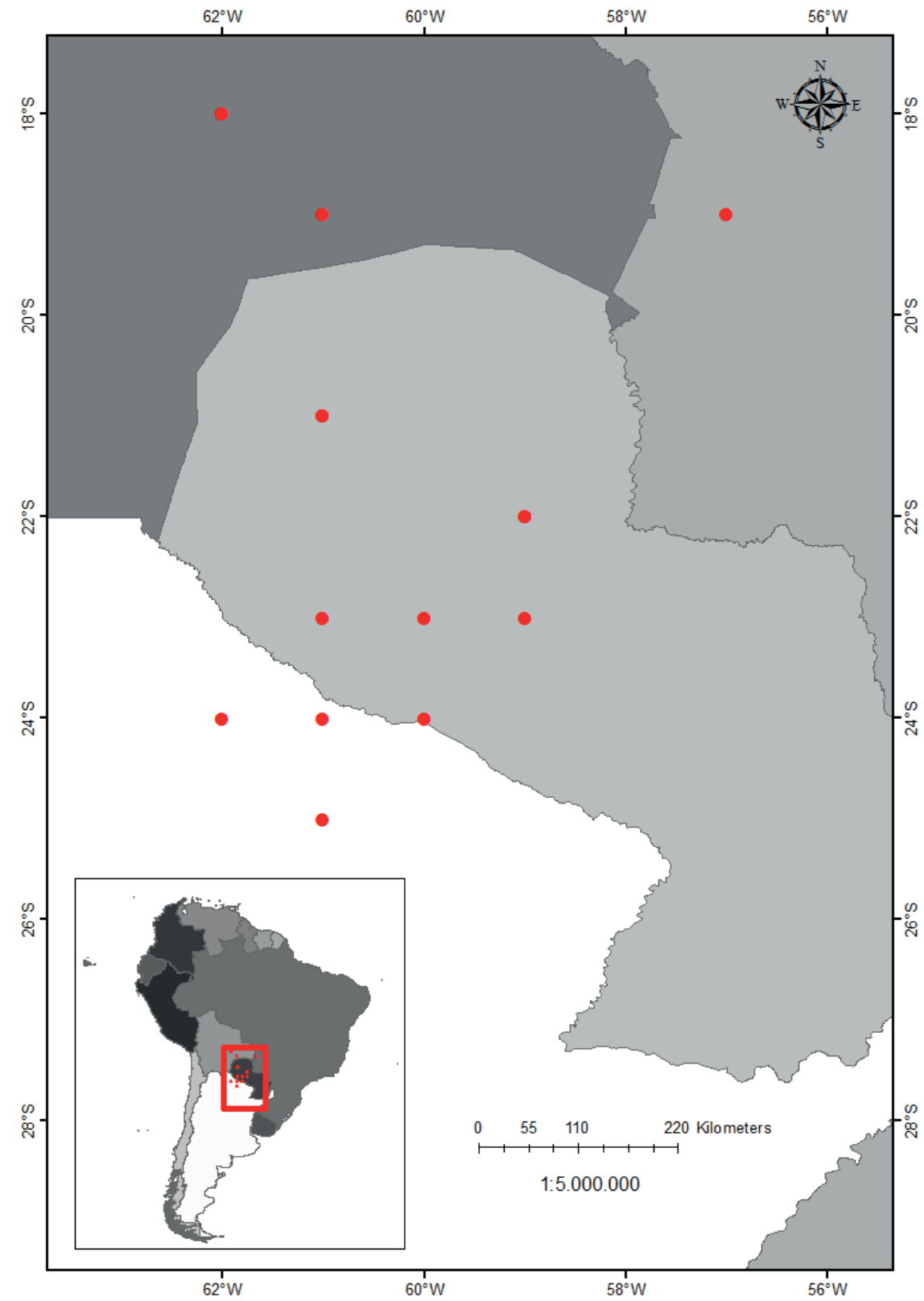

Figura 1. Distribución de Bulnesia sarmientoi a partir de datos procedentes de los herbarios de la Facultad de Ciencias Químicas de la Universidad Nacional de Asunción (FCQ), del $\mathrm{Mu}-$ seo Nacional de Historia Natural del Paraguay (PY) y de la base de datos del Missouri Botanical Garden. 
la dispersión, hábitat, distribución, abundancia, tendencia de la población, calidad de la información, amenazas, extracción, comercio ilegal, historia de la gestión, plan de gestión, régimen de cosecha, cupos, control de la cosecha, confianza de la cosecha, protección del efecto de la cosecha, efectividad de las medidas de protección estrictas y reglamentación del esfuerzo de cosecha.

Ambas etapas priorizan la información referida a la explotación comercial sobre la cual se basa la sostenibilidad, o no, de la especie.

Posteriormente se realiza la sistematización de los datos y se genera un gráfico con las respuestas, que a medida que se agregan los datos da lugar a un área central coloreada. Cuando la extracción no es perjudicial, la mayoría de las respuestas se ubican en las áreas de cautela y quedan representadas hacia el centro del círculo; por el contrario, si las respuestas ocupan áreas más periféricas del gráfico, indican la no sostenibilidad de la extracción de la especie.

\section{RESULTADOS}

En la Fig. 2 se presenta el diagrama radial con las variables ordenadas y puntuadas de acuerdo a la tabla predeterminada de la checklist (Rosser \& Haywood, 2002). Los puntos que se observan en la periferia del círculo corresponden a los criterios más preocupantes. Del estudio de la figura anterior puede deducirse lo siguiente:

1. Características biológicas: el potencial de regeneración de la especie es lento, la eficacia de la dispersión es buena en su área de distribución, la preferencia de hábitat de la especie son los bosques climácicos y no se observa mucha regeneración en bosques perturbados.

2. Condición de la Población Nacional: la especie se encuentra extendida en el territorio chaqueño, aunque actualmente está muy fragmentada. En la dinámica poblacional vemos que la tendencia de la población nacional es "reducida" pero estable. No existen cuantificaciones a nivel nacional, si bien algunos censos mencionan la abundancia, ésta varía de acuerdo al tipo de vegetación. La información sobre la especie se refiere a estudios "desactualizados".
3. Amenazas: Las principales amenazas que está enfrentando la especie son limitadas, pero no reversibles y, según el informe, estas amenazas son la sobreexplotación, y la pérdida y alteración del hábitat.

4. Gestión de la cosecha: el problema nacional de extracción ilegal o de áreas no manejadas, se declara como "pequeño" por no contar con información suficiente. La confianza en la extracción es alta debido a que se realiza solamente mediante aprobación de la autoridad de aplicación, el Instituto Forestal Nacional (INFONA), a partir del año 2003 a través de planes de manejo en propiedades privadas. Los planes de manejo son aprobados y coordinados a nivel local y nacional, la cosecha en curso es manejada bajo un marco adaptativo. La meta del régimen de cosecha en la planificación del manejo es el manejo/control de la población y maximizar el rendimiento económico; la cosecha se basa en un sistema de cupos por propiedad, no a nivel nacional ni con fundamento biológico, por lo que los cupos en curso se consideran "inciertos".

5. Control de la cosecha: el porcentaje de la cosecha legal nacional que proviene de áreas protegidas controladas por el Estado es nulo. La cosecha legal nacional proviene de fuera de las áreas protegidas, que cuentan con controles locales sobre el uso del recurso. No hay cosecha legal nacional en áreas donde no hay ningún control local firme; por lo tanto, hay una alta confianza en la gestión de la cosecha.

6. Vigilancia de la cosecha: el principal método utilizado para vigilar la cosecha es la fiscalización del plan de uso de la tierra y el principal método utilizado para vigilar los efectos de la cosecha es la estimación directa de la población. La confianza en la gestión de la cosecha es insuficiente debido a que no se cuenta con presupuesto necesario para fortalecer los controles.

7. Incentivos y beneficios de la cosecha: el uso comparado con otras amenazas es altamente negativo debido a que la especie y sus derivados son clasificados como producto de desmonte y comercializado de esta manera, lo que beneficia económicamente al productor, pero no así a la especie. No existen incentivos para la conservación de la especie a nivel nacional. Sin embargo, los incentivos para la conserva- 


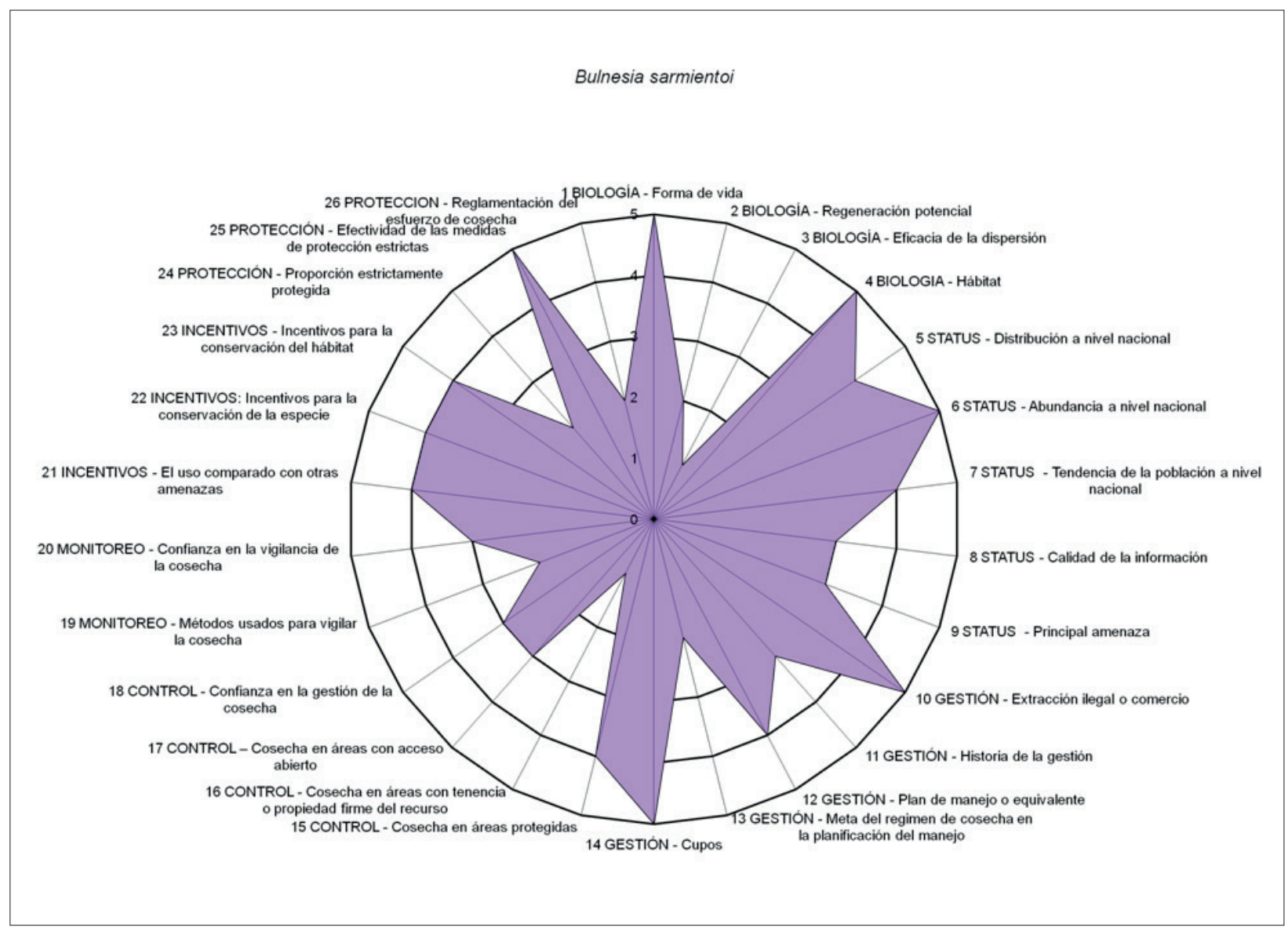

Figura 2. Visualización del estado de conservación de la especie Bulnesia sarmientoi mediante la metodología UICN para dictámenes de extracción no perjudicial (Rosser \& Haywood, 2002).

ción de su hábitat a nivel nacional se reportan como altos, a través de mecanismos como la aplicación de la Ley $\mathrm{N}^{\circ} 3001 / 2006$, de valoración y retribución de los servicios ambientales y la "venta" de carbono como stock almacenado en árboles (en el bosque o cultivados) a través de los proyectos REDD (Reducing Emissions avoiding Deforestation and Degradation); de esta manera, indirectamente, se obtiene un beneficio para todas las especies que se encuentran dentro de estos bosques.

8. Protección por efectos de la cosecha: una superficie del 5\% de cada 100 ha de desmonte donde se encuentre la especie, está excluida legalmente de la cosecha según la Resolución $\mathrm{N}^{\mathrm{o}}$ 128/03 del INFONA. La efectividad en las medidas de protección estrictas se considera incierta o nula, y sólo se conocerían con el establecimiento de un control estricto. La Reglamentación del esfuerzo de cosecha se puede considerar efectiva.

\section{DISCUSIÓN}

En la figura derivada del método Checklist to assist in making non-detriment findings for Appendix II exports aplicado a $B$. sarmientoi se puede observar que numerosos criterios están valorados en la parte periférica del gráfico, por lo que puede deducirse que la cosecha de la especie no es sostenible y, por tanto, el estado de conservación de $B$. sarmientoi podría ser preocupante. Además, la información consultada indica que las áreas boscosas en donde se encuentra la misma están siendo reemplazadas por otros sistemas productivos (principalmente agroindustriales), y que la especie, a pesar de su estatus de conservación, sigue siendo recolectada. Sus características biológicas, tales como el crecimiento extremadamente lento, contribuyen a la gravedad de la extracción, principalmente el hecho de que no existe suficiente información acerca de la dinámica natural de la especie (regeneración y mortalidad), lo que no garantiza que las poblaciones 
naturales puedan recuperarse con el régimen actual de cosecha.

A escala global, el "palo santo" está considerado como una especie en Riesgo Bajo/dependiente de conservación por la Unión Internacional para la Conservación de la Naturaleza (IUCN, 2016) en su Lista Roja de Especies Amenazadas (evaluación ya obsoleta que data de 1998, $c f$. World Conservation Monitoring Centre, 1998). Sin embargo, son varias las causas por la que B. sarmientoi en Paraguay está en riesgo de extinción local. A pesar de que la explotación comercial está regida por planes de manejo asociados a cada superficie boscosa aprobada por la autoridad de aplicación, no existe un tope comercial de volumen o densidad, establecido a nivel nacional.

Los estudios poblacionales de los que se dispone no permiten conocer el estado actual de las poblaciones de la especie. Argentina tampoco pudo recabar inicialmente toda la información necesaria para la inclusión de la misma dentro del Apéndice II, por lo que, a modo de precaución, solicitó la inclusión de la especie dentro del Apéndice III de la Convención CITES. Esta medida otorgó más tiempo a los países del área de distribución para generar información detallada sobre la especie, haciendo énfasis en los datos requeridos por la Convención. Se recuerda aquí que los Apéndices I, II y III de la Convención reflejan correctamente las necesidades de conservación de las especies, y es así que en el año 2010 Argentina presentó la propuesta de inclusión de la especie B. sarmientoi dentro del Apéndice II, con todos los requisitos necesarios que exige la CITES. En Paraguay aún no se han generado datos necesarios para la elaboración de un dictamen de extracción no perjudicial.

Se deben fortalecer los trabajos de investigación relacionados con la gestión y el monitoreo de usos de la especie. Un aspecto preocupante es la evidente debilidad en el sistema de control de la extracción y de la comercialización, no así en las condiciones necesarias para la extracción, que parecen ser favorables y correctas. Esto se debe a que la parte utilizada de la planta es la madera, por lo que se necesita un diámetro apropiado para su aprovechamiento.

Los permisos para exportar la especie son otorgados sin ninguna seguridad de que con el régimen actual de cosecha ésta sea sostenible, ya que no se realizan controles cruzados (Contraloría General de la República, 2008). En cuanto al sistema de monitoreo, no se cuenta con un método eficiente para controlar la cosecha por parte de la autoridad de aplicación, debido a la falta de presupuesto asignado para ello, según la autoridad científica de CITES en Paraguay (Céspedes, 2011); la falta de vigilancia constituye una de las debilidades del proceso.

La exportación y comercio de la madera generó un ingreso promedio de 83 millones de dólares anuales, durante los últimos 12 años (Céspedes, 2011). El incremento de las exportaciones es favorable para la economía del país; sin embargo, es preocupante desde el punto de vista de la conservación, ya que especies amenazadas incluyen el stock maderero y el "palo santo" no está excluido. Por último, el comercio ilegal de madera es frecuente en los países sudamericanos, y Paraguay no es la excepción; sólo podría combatirse con la implementación de un sistema de control regional más fortalecido, siendo necesario para ello un presupuesto acorde dentro de cada país para capacitar a los fiscalizadores y aumentar la cantidad de los mismos. Según Viveros Cabaña (2005), la comercialización ilegal es posible por la ausencia de eficientes sistemas de control y por la corrupción dentro del sector forestal, no estando los mecanismos de implementación diseñados en atención a su aplicación senci1la, resultando fomentada la burocracia y, por ende, la facilitación de la cadena de corrupción. Otra de las observaciones realizadas durante la toma de datos, que coincide con el informe del Ministerio Público, es que las cifras de exportación de la especie y sus derivados varían de una institución a otra, por lo que puede deducirse que "el marco legal y el método de control no son lo suficientemente efectivos" que deberían y que la explotación ilegal a la que se encuentra sometida la especie, más el desconocimiento técnico en manejo, no garantizan la sostenibilidad de la misma.

\section{CONCLUSIONES Y RECOMENDACIONES}

El método de evaluación cualitativa del estado de conservación de la especie utilizado en este trabajo permite una primera aproximación al estado en que se encuentra el "palo santo" en Paraguay, aunque se carece de información detallada, y ayuda a elaborar en forma preliminar un Dictamen de Extracción No 
Perjudicial de la misma. La gravedad del estado de conservación de la especie merece una mayor atención por parte de las autoridades correspondientes. Actualmente, la gestión a nivel nacional comprende la elaboración de estrategias para controlar la exportación de los productos a través de guías de identificación, la determinación de métodos más idóneos para identificar el aceite esencial y la madera, y la realización de los estudios biológicos correspondientes para establecer los cupos de exportación, que aseguren que el comercio de la especie no llevará al detrimento de la población.

Se recomienda realizar los trabajos científicos tendentes a generar datos ecológicos y poblacionales en general, incluyendo tasas de natalidad y de mortalidad, ya que únicamente los censos sobre una superficie determinada son insuficientes. Además, se deben promover políticas especiales para el "palo santo", como por ejemplo el que se realiza con una especie amenazada, regular su diámetro de corta (actualmente es de un DAP $>30 \mathrm{~cm}$ ), pues evidentemente tanto las características intrínsecas de la especie, los vacíos de información y la falta de estudios poblacionales la presentan como muy vulnerable.

Otra recomendación es la actualización de la evaluación de esta especie en la Lista Roja de la UICN, ya que se disponen de nuevos datos, de unos casi 20 años más desde la última evaluación en 1998, considerando la disminución o declive de las poblaciones y la vigencia de acuerdos legales en varios países y en CITES. Dicha evaluación sería complementaria a la establecida en el presente artículo.

\section{AGRADECIMIENTOS}

A la Lic. Biol. Juana De Egea, MSc, por la revisión del texto y la corrección en el idioma inglés.

\section{REFERENCIAS BIBLIOGRÁFICAS}

Alonso, J. \& Desmarchelier, C. 2005. Plantas medicinales autóctonas de la Argentina. Bases científicas para su aplicación en atención primaria de la salud. Literature of Latin America (L.O.L.A.), Buenos Aires.

Amat, A. \& Yajía, M. 1991. Plantas medicinales y etnofarmacología de la provincia de Misiones. Acta Farmacéutica Bonaerense 10: 153-159.

Arambarri, A. M., Novoa, M. C., Bayón, N. D., Hernández, M. P., Colares, M. N. \& Monti, C. 2011. Anatomía foliar de arbustos y árboles medicinales de la región chaqueña semiárida de la Argentina. Dominguezia 27: 1-24.

Arenas, P. 1981. Etnobotánica Lengua-Maskoy. Fundación para la Educación, la Ciencia y la Cultura, Buenos Aires.

Arenas, P. 1983. Nombres y usos de las plantas por los indígenas Maka del Chaco Boreal. Parodiana 2: 131-229.

Brack, W. \& Weik, J. H. 1994. El bosque nativo del Paraguay. Riqueza subestimada. Proyecto ALA 90-24. Serie $\mathrm{N}^{\circ} 15$. Ministerio de Agricultura y Ganadería \& Cooperación Técnica Alemana (GTZ), Asunción.

Céspedes, G. 2011. Elaboración de un Dictamen de Extracción No Perjudicial del Palo santo (Bulnesia sarmientoi Lorentz ex Griseb.) en Paraguay. Tesis de Maestría, Universidad Internacional de Andalucía, Sevilla.

CITES [Convención sobre el Comercio Internacional de Especies Amenazadas de Fauna y Flora Silvestres] 2010. Decimoquinta reunión de la Conferencia de las Partes Doha (Qatar), 13-25 de marzo de 2010. Examen de las propuestas de enmienda a los apéndices I y II. Propuesta 42. Consultado el 17 de octubre de 2016, en https://www.cites.org/ sites/default/files/esp/cop/15/prop/S-15\%20Prop-42.pdf

Contraloría General de la República 2008. Resumen Ejecutivo. Res. CGR $N^{o} 375 / 08$. Contraloría General de la República (Dirección General de Control de la Gestión Ambiental), Asunción.

Cozzo, D. 1948. Anatomía del leño de las especies argentinas de la tribu "Zygophyllae". Revista del Instituto Nacional de Investigación en las Ciencias Naturales 1: 223-361.

Degen, R. \& Mereles, F. 1996. Check-List de las plantas colectadas en el Chaco boreal, Paraguay. Rojasiana 3: 1-176.

Degen, R. \& Mereles, F. 1997. Las cortezas chaqueñas utilizadas en medicina popular. Rojasiana 4: 11-24.

Filipov, A. 1997. La farmacopea natural en los sistemas terapéuticos de los indígenas pilagá. Parodiana 10: 35-74.

Friesen Ratzlaff, V. 2004. Una guía para plantas leñosas del Chaco. Iniciativa para la Investigación y Transferencia de Tecnología Agraria Sostenible (INTTAS), Loma Plata.

Galeano, M. B. 2014. Germinación in vitro y crecimiento inicial de palo santo (Bulnesia sarmientoi Lorentz ex Griseb.) bajo estrés salino. Tesis de Ingeniería, Universidad Nacional de Asunción, Asunción.

Giménez, A. M. \& Moglia, G. 2003. Árboles del Chaco Argentino. Guía para el reconocimiento dendrológico. Secretaría del Ambiente y Desarrollo Sustentable (Ministerio de Desarrollo Social), Facultad de Ciencias Forestales (Universidad Nacional de Santiago del Estero) \& El Liberal, Santiago del Estero.

Guyra Paraguay 2014. Monitoreo ambiental del Gran Chaco americano. Informe ejecutivo de los periodos de monitoreo de los años 2011, 2012, 2013 y 2014. Guyra Paraguay, Asunción.

Hunzicker, J. H. 1980. Evolución en Bulnesia: un enfoque multidisciplinario. In: Actas del IV Congreso Latinoamericano de Genética 2. Buenos Aires: 313-324.

López, J. A., Little, E., Ritz, G., Rombold, J. \& Hahn, W. 1987. Árboles comunes del Paraguay: ñande yvyra mata kuera. Ediciones Cuerpo de Paz, Asunción.

López-Toledo, L., Burslem, D., Martínez-Ramos, M., García-Naranjo, A. 2008. Non-detriment findings report on Guaiacum sanctum in Mexico (NDF Workshop case studies, WG1 Trees, Case Study 7). CITES (Convención sobre el Comercio Internacional de Especies Amenazadas de Fauna y Flora Silvestres. Consultado el 17 de octubre de 2016, en https://cites.org/sites/default/files/ndf_material/ WG1-CS7.pdf 
Mereles, F. 2005. Una aproximación al conocimiento de las formaciones vegetales del Chaco boreal, Paraguay. Rojasiana 6: 5-48.

Mereles, F. 2014. A propósito de la distribución de Bulnesia sarmientoi Lorentz ex Griseb. (Zygophyllaceae) en el Chaco paraguayo. Revista de la Sociedad Cientifica del Paraguay 19: 187-193.

Mereles, F. \& Degen, R. 1993. Aspectos fenológicos de árboles y arbustos del Chaco boreal. I. Rojasiana 1: 49-78.

Mereles, F. \& Degen, R. 1994. Los nombres vulgares de los árboles y arbustos del Chaco boreal, Paraguay. Rojasiana 2: 67-101.

Mereles, F. \& Degen, R. 1997a. Contribución al conocimiento de los árboles y arbustos indígenas utilizados como medicinales en el Chaco Boreal (Paraguay). Parodiana 10: 75-89.

Mereles, F. \& Degen, R. 1997b. Leñosas colonizadoras e indicadoras de suelos modificados en el Chaco boreal, Paraguay. Rojasiana 4: 25-83.

Mereles, F. \& Pérez de Molas, L. 2007. Bulnesia sarmientoi Griseb. (Zygophyllaceae): estudio de base para su inclusión en el Apéndice II de la Convención CITES. Revista de la Sociedad Cientifica del Paraguay 24: 107-124.

Navarro, G. 1997. Contribución a la clasificación ecológica y florística de los bosques de Bolivia. Revista Boliviana de Ecología 2: 3-37.

Palacios, R. A. 1984. Revisión taxonómica del género Bulnesia (Zygophyllaceae). Darwiniana 25: 299-320.

Palacios, R. A. \& Mom, M. P. 2012. Zygophyllaceae. In: Rame1la, L. \& Perret, P. (Eds.), Flora del Paraguay 43. Conservatoire et Jardin Botaniques de la Ville de Genève, Genève.

Pin, A., González Salemma, G., Marín, G., Céspedes, G., Cretton, S. et al. 2009. Plantas medicinales del Jardin Botánico de Asunción. Asociación Etnobotánica Paraguaya (AEPY), Municipalidad de Asunción y Municipalidad de Ginebra, Asunción.

Rempel, E. 2003. Áreas de existencia de materia prima, usos, explotación y mercados de productos derivados de Bulnesia sarmientoi. Informe Técnico. Facultad de Ciencias Agrarias, Universidad Nacional de Asunción, Paraguay.

Rodilla, J. M. 2011. Advances in the identification and agrochemical importance of sesquiterpenoids from Bulnesia sarmientoi essential oil. Industrial Crops and Products 33: 497-503. https://doi.org/10.1016/j.indcrop.2010.10.020

Rosser, A. R. \& Haywood, M. J. (Comp.) 2002. Guidance for CITES Scientific Authorities: Checklist to assist in making non-detriment findings for Appendix II exports. IUCN, Gland \& Cambridge.

Salvat, A. E., Salvat, A., Antonacci, L., Fortunato, R. H., Suarez, E. Y. \& Godoy, H. M. 2004. Antimicrobial activity in methanolic extracts of several plant species from northern Argentina. Phytomedicine 11: 230-234. https://doi. org/10.1078/0944-7113-00327

Scarpa, G. 2000. Plants employed in traditional veterinary medicine by the criollos of northwestern Argentine Chaco. Darwiniana 38: 253-265.

Schmeda-Hirschmann, G. 1993. Magic and medicinal plants of the Ayoreos of the Chaco Boreal (Paraguay). Journal of Ethnopharmacology 39: 105-111. https://doi. org/10.1016/0378-8741(93)90025-Z

Schulz, A. G. 1997. Algunas plantas usuales del nordeste argentino. Parodiana 10: 211-241.

Tortorelli, L. 2009. Maderas y bosques argentinos 1. Orientación Gráfica Editora, Buenos Aires.

IUCN [International Union for Conservation of Nature] 2016. The IUCN Red List of Threatened Species. Version 2016-2. Consultado el 17 de octubre de 2016, en http://www.iucnredlist.org

Ulmke, C. \& August, L. 2004. Una guía para plantas nativas del Chaco Paraguayo. Iniciativas para la Investigación y Transferencia de Tecnología Agraria Sostenible (INTTAS), Filadelfia.

Viveros Cabaña, P. 2005. Gestión de recursos nativos para la forestación, reforestación y enriquecimiento de bosque de Palo Santo (Bulnesia sarmientoi) en área de distribución de la especie. Campo Ampú y Quenjacloi. USAID \& Fundación DesdelChaco, Loma Plata.

World Conservation Monitoring Centre 1998. Bulnesia sarmientoi. The IUCN Red List of Threatened Species 1998: e.T32028A9675710. Consultado el 17 de octubre de 2016, en http://dx.doi.org/10.2305/IUCN.UK.1998.RLTS. T32028A9675710.en 